\title{
Probing QCD at high energy
}

\author{
Mikko Voutilainen ${ }^{* \dagger}$ \\ University of Helsinki \\ E-mail: mikko.voutilainenecern.ch
}

\begin{abstract}
We review recent experimental work on probing QCD at high $p_{T}$ at the Tevatron and at the LHC. The Tevatron has just finished a long and illustrious career at the forefront of high energy physics, while the LHC now has its physics program in full swing and is producing results at a quick rate in a new energy regime. Many of the LHC measurements extend well into the TeV range, with potential sensitivity to new physics. The experimental systematics at the LHC are also becoming competitive with the Tevatron, making precision measurements of QCD possible.

Measurements of inclusive jet, dijet and isolated prompt photon production can be used to test perturbative QCD predictions and to constrain parton distribution functions, as well as to measure the strong coupling constant. More exclusive topologies are used to constrain aspects of parton shower modeling, initial and final state radiation. Interest in boosted heavy resonances has resulted in novel studies of jet mass and subjet structure that also test perturbative QCD predictions and parton shower models. Studies of V+jets, including heavy flavor jets, constrain important backgrounds to single top, Higgs boson searches and new physics searches. At the highest mass scales, measurements of dijet mass and dijet angular distributions are sensitive probes of new physics.
\end{abstract}

Sixth International Conference on Quarks and Nuclear Physics,

April 16-20, 2012

Ecole Polytechnique, Palaiseau, Paris

\footnotetext{
* Speaker.

${ }^{\dagger}$ For the ATLAS, CDF, CMS and D0 Collaborations.
} 


\section{Introduction}

We review the recent experimental work done on probing QCD at high $p_{T}$. The first goal of these studies is to improve our detailed description of standard model physics. The hard QCD studies presented here provide input to fitting proton parton distribution functions (PDFs), measuring strong coupling constant $\alpha_{S}$, and to studying perturbation theory (pQCD), initial and final state radiation, parton shower modeling and jet substructure.

One particular focus of the hard QCD studies is studying the gluon PDF at high Bjorken $x$, where the hadron colliders are complementary to $e$ - $p$ colliders and fixed target experiments. A second goal is to search for new physics in high $p_{T}$ events, of which an example is shown Fig. 1(a). The jet events probe the highest transverse momenta ever produced at colliders, having particular sensitivity to quark substructure, high mass resonances and contact interactions. Detailed studies of vector boson production in association with jets are also presented because these channels present irreducible background for many new physics searches, including the search for the Higgs boson.

\section{Experimental methods}

The measurements presented here have been done at the D0 and CDF experiments at the Tevatron, and at the ATLAS and CMS experiments at the Large Hadron Collider (LHC). The Tevatron has provided the experiments over $10 \mathrm{fb}^{-1}$ of proton-antiproton collisions at the centerof-mass-energy $\sqrt{s}=1.96 \mathrm{TeV}$, while the LHC has provided about $40 \mathrm{pb}^{-1}$ per experiment at $\sqrt{s}=7 \mathrm{TeV}$ in 2010, and more than $5 \mathrm{fb}^{-1}$ per experiment in 2011. The Tevatron has now stopped running, while data at the LHC is accumulating fast, as shown in Fig. 1(b).

The experimental systematics from jet energy corrections (JEC) have been steadily reduced at the LHC, and are now on par with the Tevatron experiments [1, 2, 3, 4, 5, 6] as seen in Fig. 1(c). The pile-up will be a challenge at the LHC in 2012, when the average number of simultaneous interactions increases to 30 . While pile-up is generally not a major issue for high $p_{T}$ studies, many observables will be affected. Novel theoretical and experimental approaches are available and being studied for reducing impact of pile-up.
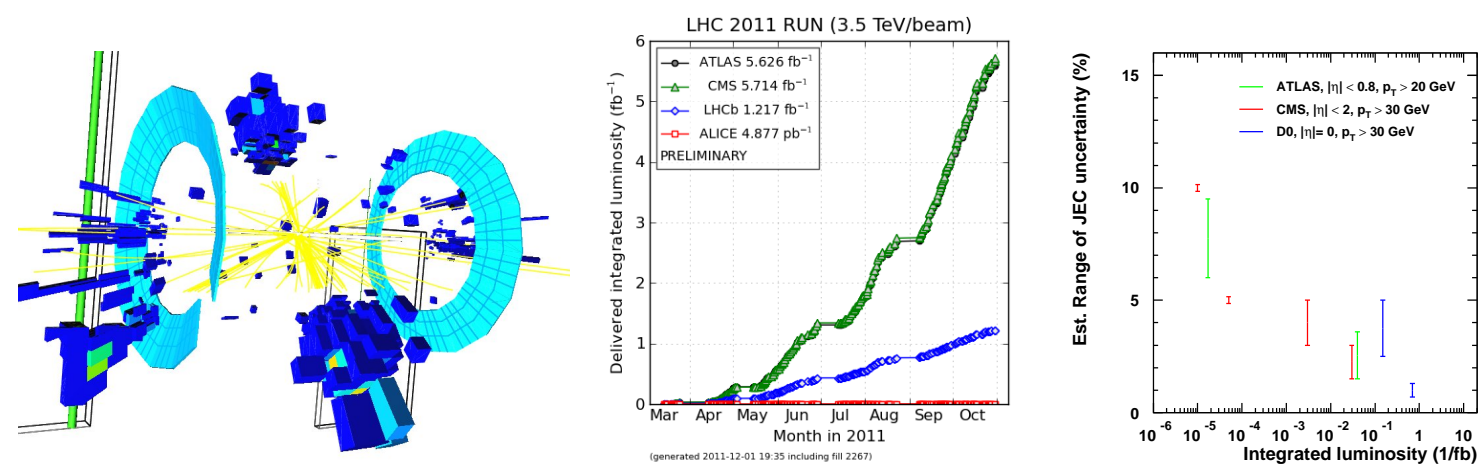

Figure 1: (a) High mass and $p_{T}$ dijet event at D0, (b) integrated luminosity at LHC in 2011, (c) estimated range of JEC systematics (courtesy of K. Rabbertz). 


\section{Constraints on PDFs and $\alpha_{S}$}

Some of the main PDF constraints from the hadron colliders have traditionally come from the inclusive jet $p_{T}$ spectrum $[6,7,8,9,10,11,12,13]$ shown in Fig.2(a) and the dijet mass spectrum $[13,14,15,16]$ measurements shown in Fig. 2(b). These measurements are particularly useful to determine the gluon PDF at high Bjorken $x$, which measurements at HERA don't constrain as well. More recently, measurements of the three-jet mass [17] shown in Fig. 2(b) have demonstrated a complementary way to decouple effects from the strong coupling constant $\alpha_{S}$ from PDF uncertainties.
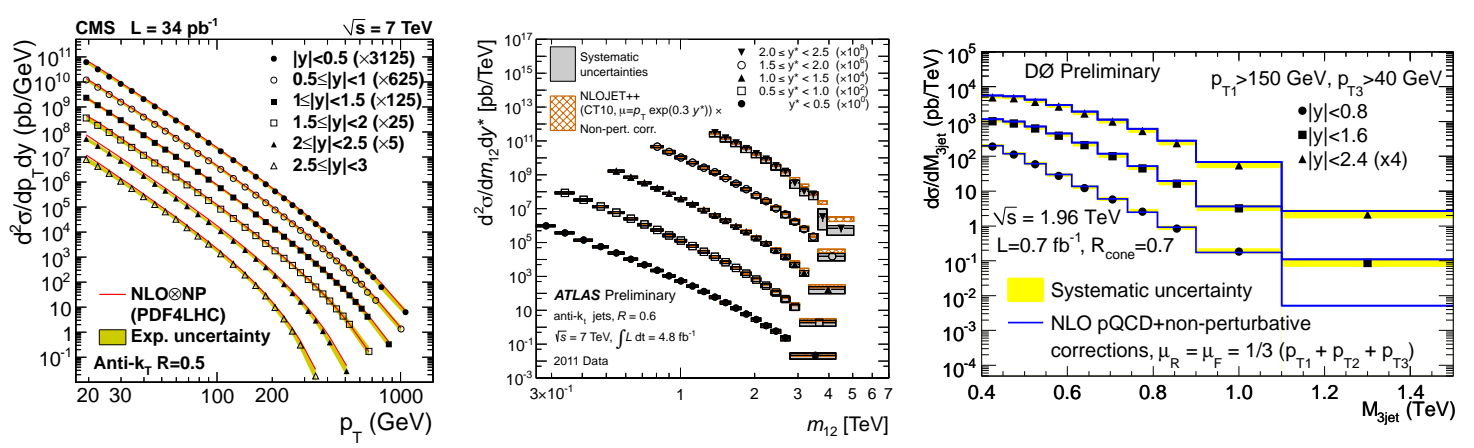

Figure 2: (a) Inclusive jet cross section, (b) dijet mass, and (c) three-jet mass cross section.

The cross section measurements show generally good agreement with the NLO predictions. Larger jet cone sizes show slight tendency for better agreement with NLO, as expected from better recovery of final state radiation. Theory calculations for a direct ratio of jet cross sections [18] indicate the effect is at the level of $5-10 \%$, consistent with observations. The experimental uncertainties at the LHC are reaching the level where the measurements can distinguish between different PDF sets [19, 20, 21, 22, 23] that are shown in Figs. 3(a,b). The measurements have some tendency to be lower than theory predictions particularly at high rapidities, as shown in Fig. 3(b).

The cross section data can be reinterpreted as a measurement of the $\alpha_{S}$ as a function of hard scale, when the $\alpha_{s}$ dependence of global PDF fits is factored out. These measurements were first done at Tevatron in Run I [24] and repeated in Tevatron Run II [25] and with the LHC data [26]. The $\alpha_{S}$ measurements show good agreement with the expected running of the coupling up to the highest scales accessible at the LHC, as shown in Fig. 3(c).


Figure 3: Inclusive jet cross section versus different PDF sets at (a) low rapidity, and (b) at high rapidity. (c) Measurement of $\alpha_{S}$ running at LHC. 


\section{Jet substructure}

At the LHC energies highly boosted heavy resonances (W, Z, top, H) decaying into quarks can be reconstructed as massive jets with a distinctive substructure. This has lead into a revival of interest in jet substructure studies [27, 28, 29]. Traditional variables include the jet mass and subjet multiplicity, shown in Figs. 4(a,b), and newer variables include subjettiness, angularity, planar flow etc. The differences between quark and gluon jets are very pronounced, which helps to separate QCD backgrounds that are dominated by gluon jets. Special algorithms for filtering jets reduce pile-up sensitivity [29], and also improve agreement between Pythia [30] and Herwig [31].

Many heavy resonances produce b-jets, and the heavy flavor production is interesting in it's own right. The inclusive b-jet cross section is $2-4 \%$ of inclusive jet cross section, and b-jets have been measured with both muon triggers and with jet triggers using secondary vertices for tagging b-jets. The measurements done at LHC [32, 33] show reasonable agreement with MC@NLO [34] predictions in Fig. 4(c), and good agreement with POWHEG+Pythia [35]. Gluon splitting is a large contribution to overall b-jet production at LHC, which may explain the differences.
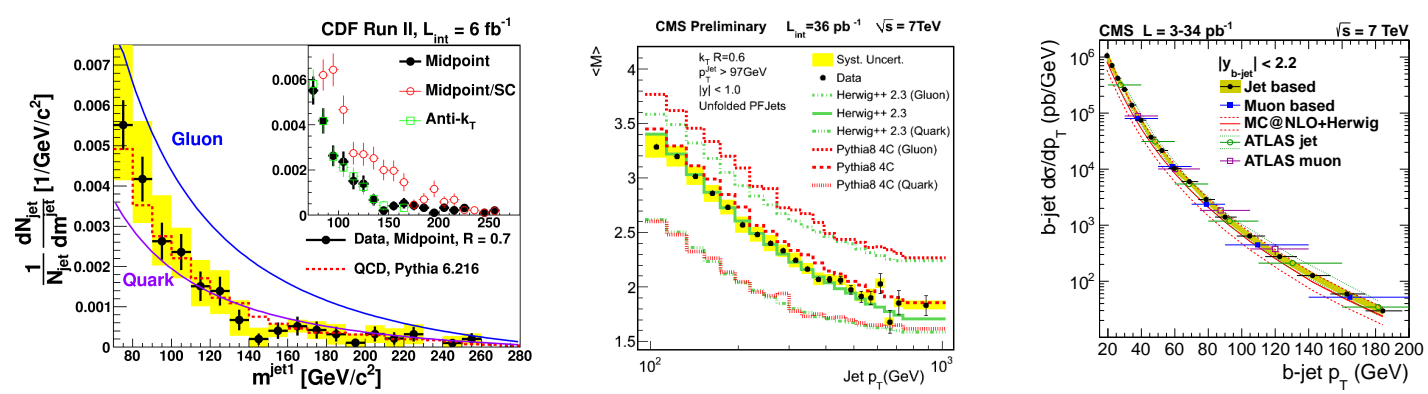

Figure 4: (a) Jet mass, and (b) subjet multiplicity. (c) Inclusive b-jet $p_{T}$ spectrum.

\section{Photons, $Z$ bosons and heavy flavor production}

The prompt photon production gives a direct handle on the interaction at the parton level, and has been proposed as a useful channel for PDF fits. Fragmentation photons that are challenging to model contribute at low $p_{T}$, but the experiments generally agree with NLO predictions $[36,37,38$, 39, 40, 41, 42], as seen in Fig. 5(a). The $\mathrm{W}$ and $\mathrm{Z}$ boson production in standard QCD processes is an irreducible background for many new physics searches so it is important to understand these processes in detail. The $\mathrm{Z}$ provides a clean signal, and the $\mathrm{Z}+\mathrm{jet}$ production has been measured versus jet $p_{T}$ as shown in Fig. 5(a), number of jets, $H_{T}$ and many other kinematic variables [43, 44, 45, 46, 47]. Comparisons with state-of-the-art calculations such as BlackHat [48] and MCFM [49] show good agreement between data and theory.

B-jets are also often produced in new physics processes, which makes associated heavy flavor production particularly interesting. The photon+heavy flavor measurements $[50,51,52,53]$ find agreement with Pythia and NLO predictions up to $p_{T}=70 \mathrm{GeV}$, as shown in Fig. 5(c). Good description of data requires higher order corrections present in Sherpa [54]. The Z+b-jet measurements $[55,56,57]$ are more limited by statistics, but find general agreement with NLO predictions. 

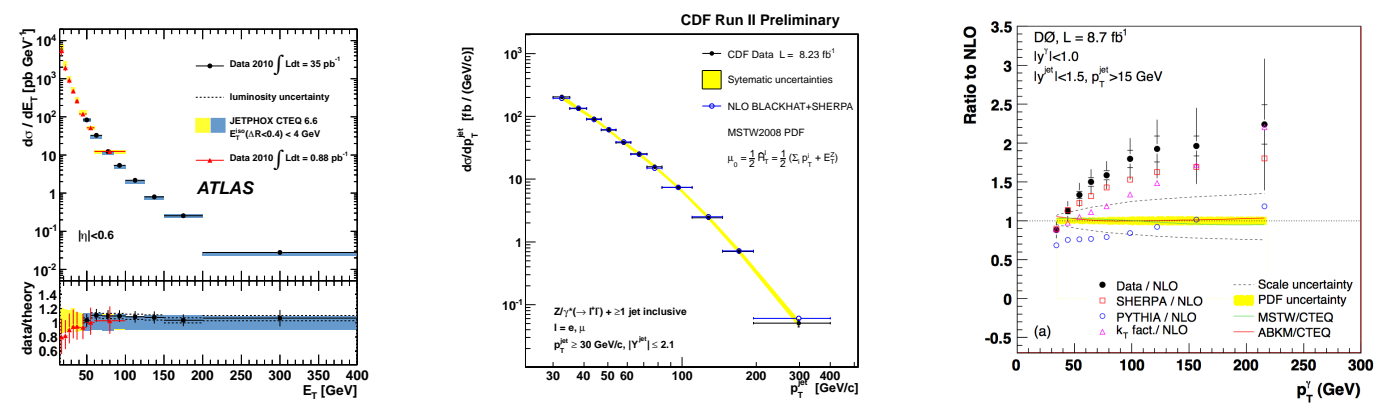

Figure 5: (a) Prompt photon production, (b) Z+jet production, (c) photon+b-jet production.

\section{Studies of event topology}

Studies of event topology are useful for testing higher order corrections and parton shower models. The measurement of diphoton azimuthal decorrelation $\Delta \phi_{\gamma \gamma}[58,59,60,61,62,63]$ has turned out to be a spectacular example of the need for next-to-next-leading order (NNLO) corrections, as shown in Fig. 6(a). Azimuthal decorrelation between pairs of jets $[64,65,66]$ is a sensitive probe of initial state radiation, and well described by NLO within PQCD applicability. It is also sensitive to multijet topologies, as shown in Fig. 6(b). Hadronic event shapes [67] probe multijet production more directly. This measurement sees good agreement with Pythia and Herwig, while MadGraph and Alpgen show some differences with data, as shown in Fig. 6(c).


Figure 6: (a) Diphoton azimuthal decorrelation, (b) dijet azimuthal decorrelation, (c) hadronic event shapes.

\section{New physics searches}

In addition to their use for constraining PDFs and backgrounds to new physics, the dijet events are used for direct searches of new physics at the highest scales accessible at hadron colliders. The studies of dijet angular distributions $[68,69]$ rely on the assumption that new physics production is isotropic and peaks at low $\chi_{\text {dijet }}=\exp \left|y_{1}-y_{2}\right|$, where $y$ is jet rapidity. The measurements are sensitive to contact interaction scales up to $\Lambda 10 \mathrm{TeV}$, but see no deviations from QCD predictions as shown in Fig. 7(a). The dijet mass spectrum measurements are also reinterpreted as searches for exotic resonances $[69,70,71]$, with highest scales reaching up to $4 \mathrm{TeV}$, as shown in Fig. 7(b). No indications of new resonances are found. 

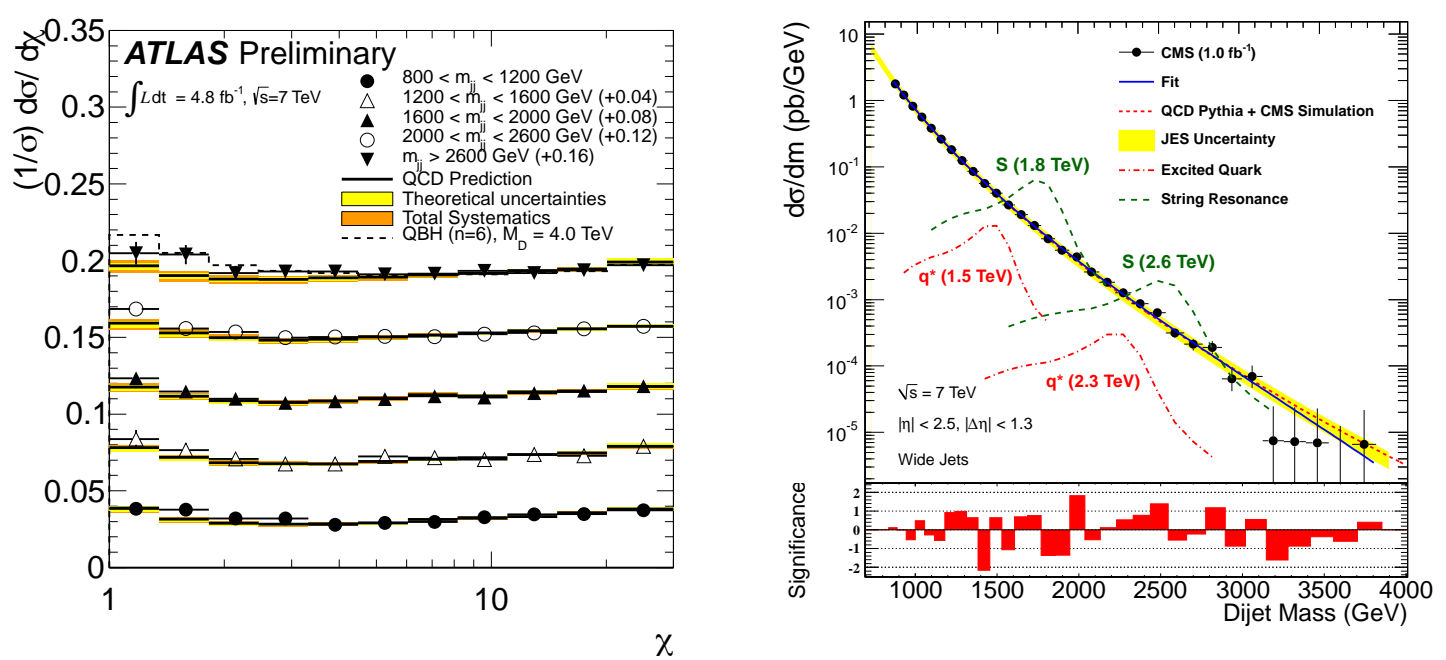

Figure 7: Search of new physics in (a) dijet angular distribution, (b) dijet mass spectrum.

\section{Conclusions and outlook}

The Tevatron finished data-taking in 2011 and the experiments are now coming out with lots of nice results from a large and well understood dataset of $10 \mathrm{fb}^{-1}$ per experiment. The LHC collected $5 \mathrm{fb}^{-1}$ per experiment in 2011 at $\sqrt{s}=7 \mathrm{TeV}$, and will continue for another $15 \mathrm{fb}^{-1}$ at $\sqrt{s}=8 \mathrm{TeV}$ in 2012. There is plenty of data and small systematics on both sides of the Atlantic, with many new results produced in the past year and more coming. The studies have generally found good agreement with NLO pQCD and recent MC tunes, with two notable exceptions: photon+heavy flavor production is not in agreement with NLO pQCD and requires higher order corrections, and diphoton azimuthal decorrelations provides a spectacular example of need for NNLO corrections. The searches for new physics are still coming out empty, but backgrounds for Higgs boson searches are under control and QCD triumphs.

\section{References}

[1] ATLAS Collaboration, CERN-PH-EP-2011-191 (2011) [hep-ex/1112.6426]. [cds]

[2] CDF Collaboration, Nucl. Instrum. Meth. A 566 (2006) 375 [hep-ex/ 05100 47]. [doi]

[3] CMS Collaboration, CMS-PAS-JME-10-003 (2010). [cds]

[4] CMS Collaboration, CMS-PAS-JME-10-010 (2010). [cds]

[5] CMS Collaboration, JINST 6 (2011) 11002 [hep-ex/1107 . 4277]. [doi]

[6] D0 Collaboration, Phys. Rev. D 85 (2012) 052006 [hep-ex/1110 . 3771]. [doi]

[7] D0 Collaboration, Phys. Rev. Lett. 101 (2008) 062001 [hep-ex/ 0802.2400 ]. [doi]

[8] CDF Collaboration, Phys. Rev. D 74 (2006) 071103 [hep-ex/ 0512020 ]. [doi]

[9] CDF Collaboration, Phys. Rev. D 75 (2007) 092006 [hep-ex/ 0701051 ]. [doi]

[10] CMS Collaboration, Phys. Rev. Lett. 107 (2011) 132001 [hep-ex/1106. 0208]. [doi] 
[11] CMS Collaboration, CMS-NOTE-2011/004 (2011).[link]

[12] CMS Collaboration, CMS-PAS-QCD-11-004 (2012). [cds]

[13] ATLAS Collaboration, CERN-PH-EP-2011-192 (2011) [hep-ex/1112.6297].

[14] CMS Collaboration, Phys. Lett. B 700 (2011) 187 [hep-ex/1104.1693]. [doi]

[15] CDF Collaboration, Phys. Rev. D 79 (2009) 112003 [hep-ex/0812 . 4036 ]. [doi]

[16] D0 Collaboration, Phys. Lett. B 693 (2010) 531 [hep-ex/1002 . 4594 ]. [doi]

[17] D0 Collaboration, FERMILAB-PUB-11-173-E (2011) [hep-ex/1104.1986].

[18] G. Soyez, Phys. Lett. B 698 (2011) 59 [hep-ph/1101.2665]. [doi]

[19] H.-L. Lai et al., Phys. Rev. D 82 (2010) 074024 [hep-ph/1 007.2241 ]. [doi]

[20] A. D. Martin et al., Eur. Phys. J. C 63 (2009) 189 [hep-ph/ 0901.0002 ]. [doi]

[21] R. D. Ball et al., Nucl. Phys. B 838 (2010) 136 [hep-ex/ 1002 . 4407 ]. [doi]

[22] H1 and ZEUS Collaboration, JHEP 01 (2010) 109 [hep-ex/0911. 0884 ]. [doi]

[23] S. Alekhin, J. Blümlein, and S. Kleinetal, Phys. Rev. D 81 (2010) 014032 [hep-ph/0908 . 2766]. [doi]

[24] CDF Collaboration, Phys. Rev. Lett. 88 (2002) 042001 [hep-ex/ 0108034 ]. [doi]

[25] D0 Collaboration, Phys. Rev. D 80 (2009) 111107 [hep-ex/ 0911.2710 ]. [doi]

[26] B. Malaescu, P. Starovoitov, CERH-PH-EP-2012-064 (2012) [hep-ph/1203.5416].

[27] CDF Collaboration, FERMILAB-PUB-11-297-E-PPD (2011) [hep-ex/1106.5952].

[28] CMS Collaboration, CMS-PAS-QCD-10-041 (2012). [cds]

[29] ATLAS Collaboration, JHEP 1205 (2012) 128 [hep-ex/1203.4606]. [doi]

[30] T. Sjöstrand, S. Mrenna, and P. Skands, JHEP 05 (2006) 026 [hep-ph / 0603175 ]. [doi]

[31] M. Bähr et al., Eur. Phys. J. C 58 (2008) 639 [hep-ph / 0803 . 0883 ]. [doi]

[32] CMS Collaboration, CERN-PH-EP-2012-036 (2012) [hep-ex/1202 . 4617].

[33] ATLAS Collaboration, Eur. Phys. J. C 71 (2011) 1846 [hep-ex/1109.6833]. [doi]

[34] S. Frixione, P. Nason, and B. R.Webber, JHEP 08 (2003) 007 [hep-ph/ 03052 52]. [doi]

[35] S. Alioli, P. Nason, C. Oleari, and E. Re, JHEP 06 (2010) 043 [hep-ph/1 002 . 2581]. [doi]

[36] D0 Collaboration, Phys. Lett. B 639 (2006) 151 [hep-ex/ 0511054 ]. [doi] [doi]

[37] D0 Collaboration, Phys. Lett. B 666 (2008) 435 [hep-ex/ 0804 . 1107 ]. [doi]

[38] CDF Collaboration, Phys. Rev. D 80 (2009) 111106 [hep-ex/ 0910 . 3623]. [doi]

[39] CMS Collaboration, Phys. Rev. Lett. 106 (2011) 082001 [hep-ex/1012.0799]. [doi]

[40] CMS Collaboration, Phys. Rev. D 84 (2011) 052011 [hep-ex/ 1108 . 204 4]. [doi]

[41] ATLAS Collaboration, Phys. Lett. B 706 (2011) 150 [hep-ex/1108.0253]. [doi]

[42] ATLAS Collaboration, Phys. Rev. D 85 (2012) 092014 [hep-ex/1203.3161]. [doi]

[43] ATLAS Collaboration, Phys. Rev. D 85 (2012) 032009 [hep-ex/1111 . 2690]. [doi] 
[44] CDF Collaboration, CDF Note 10216 (2010). [link] [pdf]

[45] CDF Collaboration, CDF Note 10394 (2011). [link] [pdf]

[46] CDF Collaboration, Preliminary $Z(l l)+$ jets results with $9.4 \mathrm{fb}^{-1}$ (2012). [link] [pdf]

[47] CMS Collaboration, JHEP 01 (2011) 010 [hep-ex/1110.3226]. [doi]

[48] C. F. Berger et al., Nucl. Phys. Proc. Suppl. 205-206 (2010) 92 [hep-ph/10 05 . 3728 ]. [doi]

[49] J. Campbell and R. K. Ellis, Phys. Rev. D 65 (2002) 113007 [hep-ph/ 0202176 ]. [doi]

[50] CDF Collaboration, Phys. Rev. D 81 (2010) 052006 [hep-ex/0 912 . 3453]. [doi]

[51] CDF Collaboration, CDF/PHYS/JET/PUBLIC/10818 (2012). [link] [pdf]

[52] D0 Collaboration, Phys. Rev. Lett. 102 (2009) 192002 [hep-ex/0901.0739]. [doi]

[53] D0 Collaboration, FERMILAB-PUB-12-082-E (2012) [hep-ex/1203.5865].

[54] T. Gleisberg et al., JHEP 0902 (2009) 007 [hep-ph/ 0811 . 4622]. [doi]

[55] D0 Collaboration, Phys. Rev. D 83 (2011) 031105 [hep-ex/1010 . 6203]. [doi]

[56] CMS Collaboration, CMS-PAS-SMP-12-003 (2012). [cds]

[57] CDF Collaboration, CDF Note 10594 (2012). [link] [pdf]

[58] D0 Collaboration, Phys. Lett. B 690 (2011) 108 [hep-ex/ 1002 . 4917]. [doi]

[59] CDF Collaboration, Phys. Rev. Lett. 107 (2011) 102003 [hep-ex/1106.5123]. [doi]

[60] CDF Collaboration,Phys. Rev. D 84 (2011) 052006 [hep-ex/110 6 . 5131]. [doi]

[61] CDF Collaboration, CDF Note 10160 (2012). [link] [pdf]

[62] ATLAS Collaboration, Phys. Rev. D 85 (2012) 012003 [hep-ex/1107. 0581 ]. [doi]

[63] S. Catani, L. Cieri, D. de Florian, G. Ferrera, M .Grazzini, ZU-TH 18/11 (2011) [hep-ex/1110.2375].

[64] D0 Collaboration, Phys. Rev. Lett. 94 (2005) 221801 [hep-ex/ 040904 0]. [doi]

[65] ATLAS Collaboration, Phys. Rev. Lett. 106 (2011) 172002 [hep-ex/1102 . 2696]. [doi]

[66] CMS Collaboration, Phys. Rev. Lett. 106 (2011) 122003 [hep-ex/1101. 502 9]. [doi]

[67] CMS Collaboration, Phys. Lett. B 699 (2011) 48 [hep-ex/1102.0068]. [doi]

[68] CMS Collaboration, CERN-PH-EP-2012-044 (2012) [hep-ex/1202 . 5535].

[69] ATLAS Collaboration, ATLAS-CONF-2012-038 (2012) [hep-ex/1103 . 3864]. [cds]

[70] CMS Collaboration, Phys. Lett. B 704 (2011) 123 [hep-ex/1107.4771]. [doi]

[71] ATLAS Collaboration, Phys. Lett. B 708 (2012) 37 [hep-ex/1108 . 6311]. [doi] 